\title{
Long-term Trend in Mean Density of Antarctic Krill (Euphausia superba) Uncertain
}

\author{
Steven G. Candy ${ }^{a^{*}}$ \\ ${ }^{a}$ SCandy Statistical Modelling Pty Ltd., 70 Burwood Drive, Blackmans Bay, TAS 7052, Australia.
}

Author's contribution

The sole author designed, analysed, interpreted and prepared the manuscript.

Article Information

DOI: 10.9734/ARRB/2021/v36i1230460

Editor(s):

(1) Dr. Paola Angelini, University of Perugia, Italy.

Reviewers:

(1) Alexander J. Werth, Hampden-Sydney College, USA. (2) Luciana Cristina Machado, Universidade de São Paulo, Brazil. Complete Peer review History, details of the editor(s), Reviewers and additional Reviewers are available here: https://www.sdiarticle5.com/review-history/76466

Method Article

Received 03 October 2021 Accepted 09 December 2021 Published 13 December 2021

\begin{abstract}
Two recent attempts to model the long-term trend in mean density of Antarctic krill in the southwestern sector of the Atlantic using the KRILLBASE dataset using different statistical methods as well as inclusion versus exclusion of data from "non-scientific" nets have resulted in disparate conclusions. The approach that used a linear mixed model (LMM) fitted to the log of mean density, after standardisation was applied to individual net hauls and with means calculated for 12 spatial strata by years between 1976 and 2016, gave a highly statistically significant linear "regional" decline north of $60^{\circ} \mathrm{S}$ and, to a lesser degree, south of this latitude. The alternative approach that used a "hurdle" model fitted to the individual net haul data, excluded regional stratification, and excluded non-scientific nets failed to detect an overall significant decline. The method of modelling log transformed means was reappraised and corrected by applying a meta-analytic LMM approach. Additionally, nonlinear smooths in year by region and a smooth in mean "climatological temperature" were included in the LMM. This model showed on average a mostly consistent decline north of $60^{\circ} \mathrm{S}$, however, neither trend was significantly different from a no-trend prediction with the trend north of $60^{\circ} \mathrm{S}$ highly uncertain. Uncertainty of predictions resulted in only weak power to detect a substantial decline of the order of $70 \%$ between 1985 and 2005. These model-based inferences neither strongly support nor reject a general hypothesis that there has been a dramatic decline in density of Antarctic krill in the Southwest Atlantic over this period.
\end{abstract}


Keywords: KRILLBASE; linear mixed models; meta-analysis; regression splines; Markov Chain Monte Carlo estimation.

\section{INTRODUCTION}

Availability of long-term (i.e. decadal) datasets of abundance estimates and subsequent estimation of year trends for the key "primary producer" of Antarctic krill (Euphausia superba) are important for understanding and quantifying recent relative to past productivity of Antarctic and sub-Antarctic ecosystems [1-3]. Important requirements for decadal and regional-scale surveys to allow unbiased estimates of year trends in abundance to be obtained are that (i) the gear and its method of application used to capture krill is efficient and that efficiency is constant over space and time using, ideally, a standardised gear/method (i.e. a single, efficient net type, haul method, time of day/night) and (ii) the total area that circumscribes the habitat is sampled comprehensively and representatively using the same survey design and sampling period (i.e. same austral summer months) each and every year of the survey. Such an ideal, multi-decadal dataset with common survey design and sampling methods which allows a finitepopulation, classical (e.g. [4]) design-based estimate (i.e. employing design-determined sample unit selection probabilities, [5]) of annual region-wide krill density is not available. What is available is KRILLBASE [6] which is a conglomeration of multi-national, multi-year surveys carried out mostly in the southwestern Atlantic ocean sector of the sub-Antarctic and Antarctic marine environments. As in Atkinson et al. [3], even when considering the modern series (i.e. 1976 to 2016, inclusive), a considerable number of different net types with varying efficiencies for in situ sampling of krill were used across surveys. In addition, there was no single, spatially-optimised sampling design for the surveys applied over these years.

As a result, empirical model-based predictions using model selection to determine the model that best approximates the data-generation process of the infinite population weighted among alternative models towards parsimony [5] is the only way to attempt to extract valid estimates of region-wide and decadal trends and their uncertainty from datasets such as KRILLBASE. In the absence of (i) and (ii) above, and employing this model-based approach, data standardisation for catch efficiency using empirical models $[2,3,6]$, and adjustment for any imbalance in spatial strata-by-year sampling intensity, using random effects for large-scale spatial strata (Atkinson et al. $[1,3]$ ), or for net haul locations or "stations" (Cox et al. [2]) must be employed. This model-based approach that includes spatial strata as random effects and holds "nuisance" covariates or factors constant in predictions [2] is commonly used to infer year trends in stock status using commercial fishinggenerated catch-per-unit-effort data $[7,8]$.

These recent efforts by Cox et al. [2] and Atkinson et al. [3] to model the long-term year trend in mean density of Antarctic krill in the southwestern sector of the Atlantic using the KRILLBASE dataset but with different statistical methods that each apply empirical model-based strategies to deal with the issues described by (i) and (ii) above have resulted in disparate conclusions. The approach of Atkinson et al. [3] used a linear mixed model (LMM) fitted to the log of mean density, after prior (i.e. external) standardisation based on empirical models using variables of depth range of haul, time of day, day of year, and net mouth area of sampling (Atkinson et al. [6]), was applied to individual net hauls and then summarised using the above mean calculated for each of 12 spatial strata by years between 1976 and 2016. This approach gave a highly statistically significant linear decline for the region defined as spatial cells north of $60^{\circ} \mathrm{S}$ and to a lesser degree for the region defined as south of this latitude. Note that it is difficult to discern from their Table 1 what the significance of the linear decline for the southern region is because the only information they present is the difference in regression slope of this region from the northern region and its statistical significance which indicated a highly statistically significant difference. The alternative approach of Cox et al. [2] used a "hurdle" model (i.e. a sub-model for presence/absence and a conditional sub-model for the log transform of non-zero haul densities which are then combined to give a single predictive model) fitted to the individual net haul data with the non-zero density response variable using the basic standardisation of number caught per square metre of the net mouth area, as given in KRILLBASE. They excluded data for "nonscientific" nets, and directly fitted the above standardisation variables (excluding net mouth area) and other covariates of depth of seabed and "climatological temperature" [6] within the hurdle model components. Cox et al. [2] failed to 
detect an overall (i.e. no regional stratification used) statistically significant decline.

In this attempt to resolve the conflict $[9,10]$ the method of modelling log transformed standardised means $[1,3]$ was reappraised using statistical theory and corrected by applying a meta-analytic LMM approach in order to more adequately model the error structure for this response variable which is a log transform of a sample statistic at the spatial strata by year level and is therefore subject to sampling error. This is in contrast to modelling the response variable measured at the lowest sampling level (i.e. individual net haul) typically applied in LMMs (e.g. [2]). Additionally, assumptions of linearity in year trends by region were investigated using low-rank thin plate regression splines [11]. Further, a covariate, or predictor variable of mean "climatological temperature", that was determined by Cox et al. [2] to be a highly significant predictor of krill density that also has a biological interpretation, was investigated as an additional term in the LMM and standardised when forming predictions of year trend. Outputs from the fit of the LMM fitted using Markov Chain Monte Carlo (MCMC) sampling included year trends and their uncertainty and predicted percentage decline between 1985 and 2005 (as used by Atkinson et al. [3]), their statistical significance, and power to detect a nominal decline of $70 \%$. Comparison, to versions of the LMM that do not consider sample statistic error as used by Atkinson et al. [3] and corresponding outputs are also given. The description of the software code using $R$ [12] and contributed libraries (MCMCglmm,Ime4,nlme) and resultant output are given in the Supplementary Material.

\section{STATISTICAL METHODS}

A corrected version of the LMM for mean standardised krill density of Atkinson et al. (2019) is given by

$$
\begin{aligned}
& \log _{10}\left(\eta_{i j}\right)=\alpha_{0}+\alpha_{1} \mathbf{I}_{(i j)}+\alpha_{2} S_{i j}+ \\
& \alpha_{3} \mathbf{I}_{(i j)} S_{i j}+\varepsilon_{i}+\tau_{i} S_{i j}+v_{j}+\xi_{i j}
\end{aligned}
$$

and

$$
\begin{aligned}
& \log _{10}\left(\bar{y}_{i j}\right)=\log _{10}\left(\eta_{i j}\right)+e_{i j} \\
& \bar{y}_{i j}=\sum_{r}^{n_{i j}} Y_{i j r} / n_{i j}
\end{aligned}
$$

where $\eta_{i j}$ is the unknown true mean standardised density for spatial cell $i$ and year $j$ observed for that cell (i.e. that obtained if a hypothetical complete census of the spatial cell could be carried out for that year) and assumed to be the expected value of $\bar{y}_{i j}$ where $\bar{y}_{i j}$ is the corresponding observed sample mean of individual haul sample standardised densities (i.e. "Standardised krill under $1 \mathrm{~m} 2$ " in KRILLBASE [3,6]), $Y_{i j r}$, from $n_{i j}$ net hauls, I as an indicator matrix with $i \times j$ rows and a single column taking the value 1 if the spatial cell $j$ is below $60^{\circ} \mathrm{S}$. Further, the error model is specified firstly using the spatial grid in the LMM using separate random effects of 'slopes and intercepts' model that allows year trend to vary across the population of grid cells. The slopes and intercept random effects were based on grid cell which gives the error term for the $i^{\text {th }}$ cell of $\varepsilon_{i}+\tau_{i} S_{i j}$ where $S_{i j}$ is the centred numeric (i.e. integer) value of year in which the $(i, f)^{\text {th }}$ survey (i.e. reassigning survey identifiers to unique identifiers within years for notational simplicity) was carried out and $\varepsilon_{i}, \tau_{i} \square \operatorname{MVN}(0, \Sigma)$ which denotes random effects as having a multivariate Gaussian distribution with expected value vector of zeros and covariance matrix with variances given by $\operatorname{diag}(\Sigma)=\left(\sigma_{\varepsilon}^{2}, \sigma_{\tau}^{2}\right)$ and covariance by $\Sigma_{12}=\Sigma_{12}=\sigma_{\varepsilon \tau}$. Other components of the error model are a random year effect (additional to Atkinson et al. [3] but included in Atkinson et al. [1]), $v_{j} \square N\left(0, \sigma_{v}^{2}\right)$, and a random lack-of-fit error, $\xi_{i j} \square N\left(0, \sigma_{\xi}^{2}\right)$ which represents departure from fixed-effect trend in $\eta_{i j}$ after accounting for the above error terms. Finally, sampling error is specified by $e_{i j} \square N\left(0, \sigma_{i j}^{2} / n_{i j}\right)$. In classical regression analysis [13] $e_{i j}$ is denoted "pure error" as distinguished from lack-of-fit error or as "measurement error" in linear mixed models [14]. Note that the usual sample estimate of each $\sigma_{i j}^{2}$ is available independently of the fit of the LMM so that these estimates can be input as a fixed (i.e assumed known) variance component in the LMM fit. The above variance for the $e_{i j}$ assumes 
that the $Y_{i j r}$ within each spatial cell by year combination are independently distributed as Gaussian, however, if they have size $n_{i j} \times n_{i j}$ covariance matrix $\Sigma_{i j}$ with possibly non-constant diagonal elements and or non-zero off-diagonal elements then $e_{i j} \square N\left(0, \mathbf{1}_{i j}^{T} \boldsymbol{\Sigma}_{i j} \mathbf{1}_{i j} / n_{i j}^{2}\right)$ where $\mathbf{1}_{i j}$ is a length $n_{i j}$ column vector of 1 's. For the following the constant variance and independence assumptions are retained but reference to this more complex error variance model is required.

A different R-software function to Ime in the nIme R-library [15] used by Atkinson et al. [3] was used here. The MCMCglmm function [16] in the library of the same name was used since the error model defined in (1) and (2) cannot be fitted using either Ime or Imer from the Ime4 R-library [17]. Note that by treating the density data for each year and spatial cell combination as a separate "experiment", then the above LMM is of general structure typically applied in metaanalyses [16]. Atkinson et al. [3] in the description of their fitted LMM, which does not use mathematical notation but simply uses software terminology, do not distinguish between lack-of-fit error, $\xi_{i j}$ (also denoted between-study random intercepts in meta-analytic LMMs), and sampling error, $e_{i j}$, variance components. Their LMM fitted to standardised sample mean krill density assumes in effect that all the $e_{i j}$ are zero combined with a definition of residual error as $\xi_{i j} \square N\left(0, \sigma^{2} / n_{i j}^{\gamma}\right)$ where $\gamma$ is a specified parameter they determined to take the value 2 from model screening using the Akaike information criterion and $\sigma^{2}$ is a modelestimated variance component assumed constant across spatial cells and years.

However, given equation (2.1), the $e_{i j}$ are not all zero but are distributed as $N\left(0, \sigma^{2} / n_{i j}^{\gamma}\right)$ where the value of $\gamma$ is known a priori from basic sampling theory, assuming constant variance and independence across stations described above, as taking the value 1 and further $\xi_{i j} \square N\left(0, \sigma_{\xi}^{2}\right)$. Atkinson et al. [3] state in the description of selection of "appropriate" variance functions for their LMM that "Model selection also identified appropriate representations of variance as a function of the reciprocal of the number of stations (from candidate fixed, power and exponential functions), to ameliorate the effects of inhomogeneity of variance". However, variance functions modelling inhomogeneity of variance typically consider variance as a function of the mean, as modelled using quasi-likelihood estimation [18], or factor-specific variances (see the LMM below), or genuine covariates (i.e as Ime was designed to model using the function varFunc; see [15]) but never as a power function of sample sizes other than the trivial case of a known power of 1 as in the above meta-analytic approach. Even under the more complex variance structure of $e_{i j} \square N\left(0, \mathbf{1}_{i j}^{T} \boldsymbol{\Sigma}_{i j} \mathbf{1}_{i j} / n_{i j}^{2}\right)$, where $\boldsymbol{\Sigma}_{i j}$ is, for example, a function of the distance between pairs of stations and unknown autocorrelation parameters, the variance cannot be simply expressed as $\sigma^{2} / n_{i j}^{\gamma}$ for any given value of $\gamma$. The other error term in equation (2.1) that can be inferred as not included in the LMM used by Atkinson et al. [3] to give the results for krill density in their Table 1 , is the term $v_{j}$ which was used as a random effect term in the LMM fitted in Atkinson et al. [1]. This is because such an error term cannot be included in an Ime fit in addition to the term $\varepsilon_{i}+\tau_{i} S_{i j}$ since Ime requires a strictly nested error structure where year as a continuous variable is nested with spatial cell. The random coefficients regression (RCR) approach (Model 1), given by equations (2.1) and (2.2), models the correlation structure in the model residuals $\xi_{i j}^{\prime}=\varepsilon_{i}+\tau_{i} S_{i j}+\xi_{i j}$ where $\operatorname{cov}\left(\xi_{i j}^{\prime}, \xi_{i k}^{\prime}\right)=\sigma_{\varepsilon}^{2}+\left(S_{i j}+S_{i k}\right) \sigma_{\varepsilon \tau}+S_{i j} S_{i k} \sigma_{\tau}^{2}$ (Equation (2.4) in [19] ; Equation (4.6.9) in [14] for which $\sigma_{\varepsilon \tau}$ is assumed to be zero) can be replaced by a $1^{\text {st }}$ order continuousautoregressive process (CAR1) also fitted using Ime (Model 2 2) where $\operatorname{cov}\left(\xi_{i j}^{\prime}, \xi_{i, j-1}^{\prime} \mid \varepsilon_{i}\right)=\sigma^{2} \varphi^{\left|S_{i j}-S_{i, j-1}\right|}$ (see Equation (5.2.7) in [14] where $\phi$ is given here by $\varphi=\exp (-\phi))$. Model 2 was compared to the RCR Model 1 and both models fitted using the weighting of $n_{i j}^{\gamma}$ with $\gamma$ fixed at 1 . The RCR 
model was also fitted using Imer, from the Ime4 library, which allowed the extra random effect term $v_{j}$ to be included along with the same weighting as the Ime fit (Model 3) and again weighting by $n_{i j}^{\gamma}$ with $\gamma$ fixed at 1 was applied. Candy et al. [20] considered the case of a CAR1 model with a common variance (i.e. $\sigma^{2}=\sigma_{i j}^{2}$ ) for measurement errors due to sampling (i.e. the $e_{i j}$ above) where these errors were not identifiable given the presence of "lack-of-fit" errors combined with a CAR1 error structure. They used Ime and gave an approximate method to deal with this lack of identifiability. This approximate method corresponds to that used in the Ime fit for Model (2). However, this approximation was not required in the metaanalytic approach fitted using MCMCglmm due to the availability of known estimates of $\sigma_{i j}^{2}$ and known fixed values of $n_{i j}$ combined with a RCR error model (see below). However, even in this case an approximation due to the $\log 10$ transformation of the means, $\bar{y}_{i j}$, was required to estimate $\hat{\sigma}_{i j}^{2}$ based on a first-order Taylor series expansion given by $\hat{\sigma}_{i j}^{2} \cong \hat{\sigma}_{(y) i j}^{2}\left[\log _{e}(10) \bar{y}_{i j}\right]^{-2}$ where $\hat{\sigma}_{(y) i j}^{2}=\left(n_{i j}-1\right)^{-1} \sum_{r}^{n_{i j}}\left(Y_{i j r}-\bar{y}_{i j}\right)^{2}$ (i.e. the usual sample estimate).

Model 1 and Model 3 were also fitted using MCMCglmm giving Model 4 and Model 5, respectively, where these last two model fits included the known estimates of sample error variances $\hat{\sigma}_{i j}^{2} / n_{i j}$ as fixed variance components in a meta-analytic approach. Additionally, for Models 4 and 5 (and Models 6 and 7) a separate lack-of-fit variance was estimated for spatial cells north of $60^{\circ} \mathrm{S}$ versus those south of this latitude (see below).

Apart from Atkinson et al.'s [3] inappropriate error model, given the sample statistic $\bar{y}_{i j}$ that they model, an even more serious limitation of their modelling effort is the failure to consider nonlinear long-term trends in density given that they restrict their consideration to linear trends. Below, a nonlinear trend model, fitted separately to each of the two regions, is considered by adding a low rank thin-plate smoothing spline in year in a penalised form by adding the term $s\left(\mathbf{I}_{(i j)}^{*}: S_{i j}, \kappa_{S}\right)$ as a 20-level random effect term (i.e. $2 \kappa_{S}$ ) to the linear terms in $S_{i j}$, as available in MCMCglmm (see [11] for expression of a lowrank thin-plate spline as a LMM). Further, a covariate term in mean climatological temperature (i.e. "Climatological temperature" in KRILLBASE), $T_{i j}$, averaged across stations within spatial cell by factor year combination is included. The variable Climatological Temperature, is described in Atkinson et al. [6] as "Long-term average February sea-surface temperature for the sampling location. This is not the actual sea temperature at the time of sampling but a climatological mean sea-surface value for February, averaged over the years 1979 to 2014". This covariate was found to be highly significant in the model for conditional density described by Cox et al. [2].

Consider the model (Model 6)

$$
\begin{aligned}
& \log _{10}\left(\bar{y}_{i j}\right)=\alpha_{0}^{\prime}+\alpha_{1} \mathbf{I}_{(i j)}+\alpha_{2} S_{i j}+\alpha_{3} \mathbf{I}_{(i j)} S_{i j}+ \\
& \alpha_{4} T_{i j}+s\left(\mathbf{I}_{(i j)}^{*}: S_{i j}, \kappa_{S}\right)+\varepsilon_{i}+\tau_{i} S_{i j}+v_{j}+\xi_{i j}+e_{i j}
\end{aligned}
$$

where

$$
\xi_{i j} \square N\left(0, \sigma_{\xi}^{2}+\mathbf{I}_{(i j)} \sigma_{N}^{2}\right) .
$$

allowing a separate lack-of-fit variance to be estimated for spatial cells north of $60^{\circ} \mathrm{S}$ (i.e. $\sigma_{\xi}^{2}+\sigma_{N}^{2}$ ) versus those south (i.e. $\sigma_{\xi}^{2}$ ) of this latitude. To fit this model using MCMCglmm we substitute sample estimates for $T_{i j}$ and $\sigma_{i j}^{2}$ of $\bar{T}_{i j}$ and $\hat{\sigma}_{i j}^{2}$, respectively. The implications of substitution of sample estimates for $T_{i j}$ due to "errors-in-variables" effects on parameter estimation is discussed later. Further, to investigate the adequacy of a linear relationship with $\bar{T}_{i j}$ given as part of the fixed model component of model (2.3) a spline term, $s\left(\bar{T}_{i j}, \kappa_{T}\right)$, was added as a random effect to give Model 7.

Cox et al. [2] note that the standardised density in KRILLBASE uses estimated regression 
parameters in its calculation and the uncertainty in these estimates was not incorporated in the error model component of the LMM in Atkinson et al. [1] and this is also the case for the LMM used by Atkinson et al. [3]. By re-running the standardisation procedure of Atkinson et al. [6] for standardisation variables of net mouth area, bottom sampling depth, day vs night sampling and days from $1^{\text {st }}$ of October using LMMs and the same KRILLBASE dataset described below, estimates of the contribution of the prediction error variance from the uncertainty due to estimation of regression parameters in the standardisation procedure were compared to the average of the $\hat{\sigma}_{i j}^{2}$ (i.e. averaged across all years by spatial strata). This is described in Supplementary Material and since this contribution was small due to the large sample size of net hauls across all years and spatial strata this source of error was not considered further. Note that Cox et al. [2] found that the contribution to the prediction error variance due to estimation error for their "standardisation" variables was of practical significance but these variables included "Water depth range (within $10 \mathrm{~km})$ " and "Climatological temperature" that were not included in the Atkinson et al. [6] standardisation.

Models 1 to 3 were compared to one another in terms of goodness of fit and parsimony using the Akikae Information Criteria (AIC) [21] while Models 4 to 7 were compared to each other using the Deviance Information Criteria (DIC) $[16,22]$. The DIC obtained from MCMCglmm is conditional on the "location parameter" [16] which are the fixed and random effect parameters. Note that it is not valid to compare Models 1 to 3 with Models 4 to 7 in terms of either AIC or DIC since Models 4 to 7 incorporate additional samplebased information (i.e. the $\hat{\sigma}_{(y) i j}^{2}$ as approximated by $\hat{\sigma}_{i j}^{2}$; see below) to that used in the fit of Models 1 to 3 . Also, Models 1 to 3 were fitted using the Maximum Likelihood (ML) option in both Ime and Imer. Note that results were very similar when Residual Maximum Likelihood (REML) [23] was maximised using the "method=REML" in Ime and "REML=TRUE" in Imer.

\subsection{Estimation and Prediction Using MCMC}

The LMM fitted in MCMCgImm is described as

$$
\mathbf{y}=\mathbf{X} \boldsymbol{\alpha}+\mathbf{Z} \boldsymbol{\beta}+\mathbf{e}^{\prime}
$$

[16] where for Model 7, $\mathrm{y}$ is the vector response variable corresponding to $\log _{10}\left(\bar{y}_{i j}\right), \boldsymbol{a}$ is the fixed effect parameters and corresponding design matrix, $\mathbf{X}, \boldsymbol{\beta}$ is the random effect parameter vector $\boldsymbol{\beta}=(\boldsymbol{\varepsilon}, \boldsymbol{\tau}, \mathbf{v}, \mathbf{e} . .$.$) augmented by$ the random effects determining the nonlinear contributions to the thin-plate spline terms, and the corresponding design matrix, $\mathbf{Z}$, and $\mathbf{e}^{\prime}$ is the residual error term with elements $\xi_{i j}$.

The default priors [16] for the fixed effect parameters $\alpha$ in MCMCglmm were used (i.e. independent Gaussian with expected value of zero and large variance of $e^{10}$ ).

Priors for the variance structures in MCMCgImm (i.e. described as $\mathbf{R}=\operatorname{var}\left(\mathbf{e}^{\prime}\right)$ and $\mathbf{G}=\operatorname{var}(\boldsymbol{\beta})$ structures) were defined by the expected variance ("V") and degree of belief parameter ("nu") for independent univariate inverse-gamma distributions for all variance parameters with these two parameters set to 1 and 0.002 , respectively [16]. The prior for the variance of $\mathrm{e}$ was set to 1 and subsequently fixed at 1 during estimation with corresponding diagonal elements of $\mathbf{Z}$ set to $\hat{\sigma}_{i j} / \sqrt{n_{i j}}$, or equivalently, the mev option of MCMCglmm was set to $\hat{\sigma}_{i j}^{2} / n_{i j}$.

MCMC sampling involved 130,000 draws from the posterior distribution for the full parameter set $\boldsymbol{\theta}$ where $\boldsymbol{\theta}=\left(\boldsymbol{\theta}_{1}, \boldsymbol{\theta}_{2}, \boldsymbol{\theta}_{3}\right)$ and where $\boldsymbol{\theta}_{1}=\boldsymbol{\alpha}$, $\mathbf{G}=\mathbf{G}\left(\boldsymbol{\theta}_{2}\right)$, and $\mathbf{R}=\mathbf{R}\left(\boldsymbol{\theta}_{3}\right)$. A "burn-in" phase of 30,000 and "thinning rate" of 1 in 100 was used, giving a final sample of 1000 values. The thin-plate spline fitted in Models 6 and 7 separately for each of the two spatial strata of north and south of the below $60^{\circ} \mathrm{S}$ latitude was fitted with number of knots set at the default at

$\kappa_{S}$ of 10 for each strata and fitted as a penalised smoother [16] by including the spline term $s\left(\mathbf{I}_{(i j)}^{*}: S_{i j}, \kappa_{S}\right)$ in the random component of the MCMCglmm function call. Similarly, the spline in Climatological Temperature for Model 7 was 
fitted as a random effect term with ${ }^{\kappa_{T}}$ also set at the default of 10 .

The posterior distribution of $\theta$ obtained in the fit of Models 4 to 7 is given by $f(\boldsymbol{\theta}) \prod_{i j} \ell\left\{\boldsymbol{\theta} \mid \bar{y}_{i j}, \hat{\sigma}_{(y) i j}^{2}\right\}$ where $f(\boldsymbol{\theta})$ is the prior density function and $\ell\left\{\boldsymbol{\theta} \mid \bar{y}_{i j}, \hat{\sigma}_{(y) i j}^{2}\right\}$ is the likelihood. The $\bar{y}_{i j}$ and $\hat{\sigma}_{(y) i j}^{2}$ are jointly the sufficient statistics for parameters $\eta_{i j}$ and $\sigma_{(y) i j}^{2}$ given $Y_{i j r} \square N\left(\eta_{i j}, \sigma_{(y) i j}^{2}\right)$ so the above likelihood contains all the information in the haullevel data on these parameters given these distributional assumptions. Therefore, it is important to note the estimation used in Ime and Imer fits does not incorporate the sufficient statistics $\hat{\sigma}_{(y) i j}^{2}$ (or their approximation $\hat{\sigma}_{i j}^{2}$ ) and therefore the parameter estimates and the AIC statistic obtained with $\bar{y}_{i j}$ as the dependent variable and ignoring these other sufficient statistics does not incorporate all the information in the haul-level data whereas the meta-analysis approach using MCMCgImm does. The above arguments would be expected to hold approximately if as assumed in the above LMMs that $\log _{10}\left(\bar{Y}_{i j}\right)$, with sample realisation $\log _{10}\left(\bar{y}_{i j}\right)$, is Gaussian distributed rather than $Y_{i j r}$ and therefore $\bar{Y}_{i j}$.

Predictions of year trends from Model 7 for the two regions while controlling for mean Climatological Temperature, $T_{i j}$, was obtain for the $r^{\text {th }}$ MCMC sample by

$$
\hat{\mathbf{y}}_{r}^{*}=\log _{10}\left\{\hat{\eta}\left(\mathbf{S}_{N}, \mathbf{S}_{S}\right)\right\}=\mathbf{X}^{*} \hat{\boldsymbol{\alpha}}_{r}+\mathbf{Z}^{*} \hat{\boldsymbol{\beta}}_{r}^{*}
$$

where $S_{N}$ is the (vector) set of years populated by values of mean density in the dataset for the northern region (i.e. north of $60^{\circ} \mathrm{S}$ ) and similarly for $\mathbf{S}_{S}$ for the southern region, while $\mathbf{X}^{*}$ has first four columns corresponding to the year fixed effect terms of $\left(\mathbf{1}_{N}, \mathbf{1}_{S}\right),\left(\mathbf{0}_{N}, \mathbf{1}_{S}\right),\left(\mathbf{S}_{N}, \mathbf{S}_{S}\right)$, and $\left(\mathbf{0}_{N}, \mathbf{S}_{S}\right)$, where subscripts define the length of vectors corresponding to year vectors and the final column is given by $\left(\overline{\mathbf{T}}_{N}, \overline{\mathbf{T}}_{S}\right)$ where mean values of Climatological Temperature averaged over all years and spatial cells within each region have been appropriated replicated. A similar process was used to derive $\mathbf{Z}^{*}$ by selecting elements from the 20 columns $\left(\kappa_{S}=10\right.$ for each region) of $\mathbf{Z}$ corresponding to the model term $s\left(\mathbf{I}_{(i j)}^{*}: S_{i j}, \kappa_{S}\right)$ and averaging across years within each region for each of the $10\left(\kappa_{T}=10\right)$ columns of $\mathbf{Z}$ corresponding to the model term $s\left(\bar{T}_{i j}, \kappa_{T}\right)$. Obtaining predictions only for "design" values for $\mathbf{X}$ and $\mathbf{Z}$ using the above simple process rather than using the analytical expression to obtain nonlinear interpolation between knot values for the spline terms (i.e. Equation (2) of [11]) in order to graphically study smoothed year trends circumvents the lack of such a facility for the latter in MCMCglmm and is adequate since there are few missing years within the year ranges for each region. Median and $90 \%$ quantile values for each year by region using the set of 1,000 values of the $\hat{\mathbf{y}}^{*}$ vector were obtained by simple summarisation of this MCMC sample.

As described above, mean Climatological Temperature, $T_{i j}$, averaged across stations within spatial cell by factor year combination was used as a covariate (i.e. predictor variable). This covariate was found, at the individual net haul level, to be highly significant in the model for conditional density described by Cox et al. [2]. For predictions of long-term trend in log density, Climatological Temperature was controlled for (i.e. predictions standardised) by setting its value to average values for north and south strata, respectively, given the justification in Cox et al. [2] (see Discussion).

\subsection{Prediction of Percentage Change in Average Density between Reference Years}

The percentage change in average density between years $S$ and $S+\Delta S, P(S, \Delta S)$, for spatial cells above $60^{\circ} S$ using the linear model (1) does not depend on $S$ but only on $\Delta S$ since it is given by 


$$
\begin{aligned}
& P(S, \Delta S)=100\left\{\frac{\eta(S+\Delta S)}{\eta(S)}-1\right\}=100 \\
& {\left[\exp \left\{\log _{e}(10) \alpha_{2} \Delta S\right\}-1\right]}
\end{aligned}
$$

This follows from the very simple differential equation

$$
\frac{1}{\eta(S)} \frac{d \eta(S)}{d S}=\alpha_{2} \log _{e}(10)
$$

Atkinson et al. [3] used the same fixed effect terms as model (1) and applied an unweighted average of the estimates of the regression slope over the two regions to calculate the percentage change in mean density. The value of $P(S, \Delta S)$ in this case is

$$
P(S, \Delta S)=100\left[\exp \left\{\begin{array}{l}
\log _{e}(10) \\
\left(\alpha_{2}+0.5 \alpha_{3}\right) \Delta S
\end{array}\right\}-1\right]
$$

, noting that the $\mathrm{R}$ default contrasts for unordered factors gives $\alpha_{3}$ as a difference in slope, while the standard error of the estimate after substituting regression parameters with their estimates is approximately (i.e. using a $1^{\text {st }}$ order Taylor series approximation)

$$
\begin{aligned}
& \operatorname{se}\{\hat{P}(S, \Delta S)\} \cong \Delta S \log _{e}(10) \\
& \{\hat{P}(S, \Delta S)+100\}\left(\mathbf{c}^{T} \boldsymbol{\Sigma}_{\hat{\alpha}} \mathbf{c}\right)^{1 / 2}
\end{aligned}
$$

where $\Sigma_{\hat{\alpha}}$ is the variance-covariance matrix just for the regression slope parameters and $\mathbf{c}^{T}=(1,0.5)$. The estimate of $\Sigma_{\hat{\alpha}}$ was obtained as either Ime or MCMCgImm output. Using the estimates in Atkinson et al. [3] (their Table 1) of $\left(\alpha_{2}, \alpha_{3}\right)$ of $(-0.065,0.044)$ the estimate of $\hat{P}(S, \Delta S)$ over close to two decades (i.e. $\Delta S$ $=20.5$ ) gives an $87 \%$ decline and not the $70 \%$ value that they reported. Note also that Atkinson et al. [3] do not present any uncertainty bounds on their estimate of a $70 \%$ decline.
For Models 6 and 7 given the nonlinear trend with $S$ using thin-plate spline terms, the estimate of $P(S, \Delta S)$ is not such a simple function of $\Delta S$ but requires prediction of $\hat{\eta}(S)$ at both $S$ and $S+\Delta S$. The MCMC sample of parameters in Models 6 and 7 obtained using MCMCglmm was used to obtain predictions of $\hat{P}(S, \Delta S)$ and the log-ratio, $L_{R}(S, \Delta S)=\log _{e}\{\hat{\eta}(S+\Delta S) / \hat{\eta}(S)\}$, along with the standard errors, probability levels (i.e. Type I error) for the null hypothesis $\left(H_{0}\right)$ that $L_{R}(S, \Delta S)=0$, and the power [i.e. 1 Prob(Type II error)] to detect a $70 \%$ or greater decline [i.e. $H_{1}$; the alternative hypothesis, $L_{R}(S, \Delta S) \leq \log _{e}(0.30)$ ] for each region.

\section{DATA}

Table 1 shows the criteria for the selection of the subset of the full KRILLBASE dataset [6] used for modelling which was designed to match the selection criteria described by Atkinson et al. [3]. The final dataset consisted of 7328 records for individual hauls which were subsequently used to calculate the means of standardised density for spatial cell by year combinations (i.e. $\bar{y}_{i j}$ ). There were 12 spatial strata or cells constructed as described in Table 1 which had at least 50 stations sampled when totalled across all years. There were 291 combinations of spatial cell by year populated with at least one haul (i.e. $n_{i j}>0$ ), but this was further reduced to 268 combinations for which $n_{i j}$ was two or greater so that $\hat{\sigma}_{i j}^{2}$ could be calculated. Of these 268 means, 207 were for the south of $60^{\circ} \mathrm{S}$ strata and 61 for the north of $60^{\circ} \mathrm{S}$ strata while the corresponding total hauls per strata were 6145 and 1083, respectively. The subset of KRILLBASE used by Cox et al. [2] that was restricted to "scientific" net types of "IsaacsKidd", "RMT8", and "2 m fixed- frame net" gave a total over both strata of 5,962 net hauls (including zero-catch hauls). 
Table 1. Data subsetting of KRILLBASE

\begin{tabular}{|c|c|c|}
\hline Selection Criterion & $\begin{array}{l}\text { Number of } \\
\text { Records } \\
\text { Retained }\end{array}$ & $\begin{array}{l}\text { Comments } \\
\text { (data selection as described in } \\
\text { Atkinson et al. [3] }\end{array}$ \\
\hline Years $^{\mathrm{a}} 1976$ to 2016 inclusive & 11,090 & $\begin{array}{l}\text { Austral summers }{ }^{\mathrm{b}} \text {, earliest record: } 19 \\
\text { Nov } 1975\end{array}$ \\
\hline Remove winter records & 10,920 & $\begin{array}{l}\text { retain stations sampled after } 1^{\text {st }} \text { October } \\
\text { and before } 1^{\text {st }} \text { May }\end{array}$ \\
\hline $\begin{array}{l}\text { Latitudinal range } 70^{\circ} \mathrm{S} \text { to } 50^{\circ} \mathrm{S} \text { inclusive } \\
\text { Longitudinal range } 80^{\circ} \mathrm{W} \text { to } 20^{\circ} \mathrm{W} \text { inclusive }\end{array}$ & 8,055 & $\begin{array}{l}\text { Spatial cells corresponding to } 2.5^{\circ} \\
\text { latitudinal zones further subdivided into } \\
\text { shelf and oceanic zones }\end{array}$ \\
\hline Variable "No. of krill under $1 \mathrm{~m}^{2 \text { " non-missing }}$ & 7,912 & \\
\hline $\begin{array}{l}\text { Variable "Bottom sampling depth }(\mathrm{m}) \text { " }=\text { or > } \\
50 \mathrm{~m}\end{array}$ & 7,777 & \\
\hline \multicolumn{3}{|c|}{ Variable "Top sampling depth $(\mathrm{m})$ " $=$ or $<20 \mathrm{~m} 7,352$} \\
\hline $\begin{array}{l}\text { Remove data for spatial cells }{ }^{\mathrm{c}} \text { with }<50 \\
\text { stations }\end{array}$ & 7,328 & $\begin{array}{l}\text { At least } 50 \text { stations sampled in total over } \\
\text { all years }\end{array}$ \\
\hline
\end{tabular}

For predictions of long-term trend in log density, Climatological Temperature was controlled for (i.e. predictions standardised) by setting its value to average values of $3.05^{\square} \mathrm{C}$ and $0.75^{\square} \mathrm{C}$ for north and south strata, respectively, as described above.

\section{RESULTS}

Fig. 1 shows the mean densities used in model fits along with bars corresponding to twice the standard error (i.e. $\hat{\sigma}_{i j} / \sqrt{n_{i j}}$ ) above and below the mean for each of the spatial strata with panels representing the latitudinal component and filled versus unfilled symbols corresponding to shelf versus oceanic depth strata, respectively. Fitted splines specific to each strata are shown within panels combined with shelf (solid lines) and oceanic depth strata (dashed lines). These splines were obtained from the fit of Model 6 using MCMCglmm but with the term $\alpha_{4} T_{i j}$ dropped and the 2-level regional factor replaced by the 12-level (fixed effect) spatial cell factor (requiring the random intercept and slope term to be dropped). Fig. 1 can be compared directly to Fig. 2(a) in Atkinson et al. [3] and generally shows good agreement apart from there being only three means for the $50-52.5^{\circ} \mathrm{S}$ (oceanic) strata in Fig. 1 here versus 10 means in their Fig. 2(a). The R-code used to construct the dataset used for model fitting and how this data was selected to correspond to that described in Atkinson et al. [3] and corresponding output is given in Supplementary Material so that the results given here can be validated.

Table 2 gives the fit of the seven LMMs (Models 1 to 7) considered here, two fitted with Ime, one with Imer, and four fitted with MCMCgImm.

Table 3 gives the estimate of $\hat{P}(S, \Delta S)$ by region for Models 1 to 4 which are strictly linear in centred year $S$. Table 3 also gives estimates of the log-ratio of predicted mean density for each region for the 2005 estimate as a ratio of the 1985 estimate corresponding to the approximate midpoint of each of the two periods that Atkinson et al. [3] denote as "the first and second halves (1976-1995) and (1996-2016) of the modern era". The 20-year period between these midpoints corresponding closely to the 20.5-year period that they apply to arrive at a percentage reduction, $\hat{P}(S, \Delta S)$ of $70 \%$. They do not give their method of calculation to arrive at this value but as mentioned earlier this value is not the correct value of $\hat{P}(S, \Delta S)$. Using the MCMC sample of Model 6 parameters to obtain the corresponding set of predictions, hypothesis tests and power calculations are also given in Table 3.

Fig. 2 shows the means for spatial cells (i.e. across years within spatial cell) for Climatological Temperature, $\bar{T}_{i}$, versus mid-latitude for each spatial cell along with corresponding double standard error (SE) bars. 
Table 2. LMM parameter estimates fitted to KRILLBASE standardised density

\begin{tabular}{|c|c|c|c|c|c|c|c|c|c|}
\hline \multirow{2}{*}{$\begin{array}{l}\text { Model } \\
\text { (R-function) } \\
\text { AIC or DIC }\end{array}$} & \multicolumn{3}{|c|}{ Year Trend (SE or $95 \%$ CLs) } & \multirow{2}{*}{$\begin{array}{l}\text {-Mean Temp } \hat{\alpha}_{4} \\
\text { (SE or } 95 \% \text { CLs) } \\
\quad s\left(\bar{T}_{i j}, 10\right) ; \text { Model } 7\end{array}$} & \multirow{2}{*}{$\hat{\sigma}_{\varepsilon}$} & \multirow{2}{*}{$\begin{array}{l}\hat{\sigma}_{\tau} \\
(\hat{\varphi} \text { for } \\
\text { Model 2) }\end{array}$} & \multirow{2}{*}{$\hat{\sigma}_{v}$} & \multirow{2}{*}{$\hat{\sigma}_{\xi}, \hat{\sigma}_{N}$} & \multirow{2}{*}{$\begin{array}{l}\hat{\sigma} \\
\text { or } \\
\text { fixed }^{\mathrm{c}} \text { at } \hat{\sigma}_{i j}\end{array}$} \\
\hline & $\begin{array}{l}\text { North, } \hat{\alpha}_{2} \\
\text { (SE or } 95 \% \\
\text { CLs) }\end{array}$ & $\begin{array}{l}\text { South }^{\mathrm{a}} \hat{\alpha}_{3} \\
\text { (SE or } 95 \% \\
\text { CLs) }\end{array}$ & $\begin{array}{l}s\left(\mathbf{I}_{(i j)}^{*}: S_{i j}, 10\right)_{\mathrm{b}} \\
(95 \% \mathrm{CLs})\end{array}$ & & & & & & \\
\hline $\begin{array}{l}\text { (Ime) } \\
\text { AIC=678.3 }\end{array}$ & $\begin{array}{l}-0.0546 \\
(0.0101)\end{array}$ & $\begin{array}{l}0.0341 \\
(0.0111)\end{array}$ & - & - & 0.1989 & $2.4406 \mathrm{e}-06$ & - & - & 3.2572 \\
\hline $\begin{array}{l}2 \text { (Ime) } \\
\text { AIC }=674.0\end{array}$ & $\begin{array}{l}-0.0557 \\
(0.0106)\end{array}$ & $\begin{array}{l}0.0351 \\
(0.0118)\end{array}$ & - & & 0.1617 & 0.1255 & - & - & 3.2838 \\
\hline $\begin{array}{l}3 \text { (Imer) } \\
\text { AIC=649.5 }\end{array}$ & $\begin{array}{l}-0.0432 \\
(0.0109)\end{array}$ & $\begin{array}{l}0.0213 \\
(0.0104)\end{array}$ & - & & 0.2428 & $4.8700 \mathrm{e}-04$ & 0.3222 & - & 2.8205 \\
\hline $\begin{array}{l}4(\mathrm{MCMCglmm}) \\
\mathrm{DIC}=561.0\end{array}$ & $\begin{array}{l}-0.0453 \\
(-0.0924 \\
-0.0051)\end{array}$ & $\begin{array}{l}0.0360^{\text {ns }} \\
(-0.0165 \\
0.0876)\end{array}$ & - & - & 0.4366 & 0.0362 & 0.3090 & $\begin{array}{l}0.5134 \\
0.9040\end{array}$ & fixed \\
\hline $\begin{array}{l}5(\mathrm{MCMCglmm}) \\
\mathrm{DIC}=545.7\end{array}$ & $\begin{array}{l}-0.0450^{\text {ns }} \\
(-0.0917 \\
0.0031)\end{array}$ & $\begin{array}{l}0.0420^{\text {ns }} \\
(-0.0105 \\
0.0965)\end{array}$ & - & $\begin{array}{l}-0.4496 \\
(-0.6474,-0.2221)\end{array}$ & 0.5860 & 0.0378 & 0.2629 & $\begin{array}{l}0.4951 \\
0.9133\end{array}$ & fixed \\
\hline $\begin{array}{l}6(\mathrm{MCMCglmm}) \\
\mathrm{DIC}=542.4\end{array}$ & $\begin{array}{l}-0.0279^{\text {ns }} \\
(-0.3365 \\
0.2604)\end{array}$ & $\begin{array}{l}0.0147^{\text {ns }} \\
(-0.4257 \\
0.3820)\end{array}$ & $\begin{array}{l}0.0005 \\
(0.0001,0.0013)\end{array}$ & $\begin{array}{l}-0.4795 \\
(-0.7481,-0.2829)\end{array}$ & 0.6086 & 0.0388 & 0.2883 & $\begin{array}{l}0.4875 \\
0.8815\end{array}$ & fixed \\
\hline $\begin{array}{l}7(\mathrm{MCMCglmm}) \\
\mathrm{DIC}=536.0\end{array}$ & $\begin{array}{l}-0.0282^{\text {ns }} \\
(-0.3155 \\
0.2943)\end{array}$ & $\begin{array}{l}0.0163^{\text {ns }} \\
(-0.3916 \\
0.4115)\end{array}$ & $\begin{array}{l}0.0005 \\
(0.0001,0.0013)\end{array}$ & $\begin{array}{l}-0.6156^{\mathrm{ns}} \\
(-1.3197,0.2583) \\
0.0259 \\
(0.0003,0.0865)\end{array}$ & 0.5387 & 0.0368 & 0.3113 & $\begin{array}{l}0.4762 \\
0.8933\end{array}$ & fixed \\
\hline
\end{tabular}

${ }^{b}$ Expressed here as a variance of the corresponding 20 (i.e. 10 per strata) random effect estimates. Note that the contribution to predictions is the vector of random effect estimates multiplied by the corresponding columns of the Z matrix (Hadfield, 2010) so that graphical output is more informative than this variance in quantifying departures from linearity.

\footnotetext{
${ }^{c}$ Fixed variances are $\hat{\sigma}_{i j}^{2} / n_{i j}$ in MCMCgImm while Ime weights via varPower and Imer weights via weights are $1 / \sqrt{n_{i j}}$ giving variances of $\hat{\sigma}^{2} / n_{i j}$. ${ }^{*} P<0.05 ;{ }^{* *} P<0.01 ;{ }^{* * *} P<0.001 ;{ }^{n s} P>0.05$.
} 
Table 3. Percentage change in mean density between midpoint of periods 1976-1995 and 1996-2016 obtained from LMM parameter estimates for North and South of $60^{\circ} \mathrm{S}$ strata

\begin{tabular}{|c|c|c|c|c|c|}
\hline \multirow[t]{2}{*}{$\begin{array}{l}\text { Model } \\
\text { (R-function) }\end{array}$} & \multicolumn{3}{|c|}{$\begin{array}{c}\text { Percentage Change in Mean Density } \hat{P}(\Delta S=20.5) \\
\text { (SE) }\end{array}$} & \multicolumn{2}{|c|}{$\begin{array}{l}\text { Ratio of predicted mean densities } \\
\left(L_{R}\left(1985^{c}, 20\right), \text { SE, Power }\right.\end{array}$} \\
\hline & North & South & Average $^{a}$ & North & South \\
\hline 1 (Ime) & $\begin{array}{l}-92.4 \\
(3.6) \\
\end{array}$ & $\begin{array}{l}-62.1 \\
(8.4)\end{array}$ & \multicolumn{3}{|l|}{$\begin{array}{l}-83.0 \\
(4.4)\end{array}$} \\
\hline 2 (Ime) & $\begin{array}{l}-92.8 \\
(3.6)\end{array}$ & $\begin{array}{l}-62.2 \\
(9.0)\end{array}$ & \multicolumn{3}{|l|}{$\begin{array}{l}-83.5 \\
(4.5)\end{array}$} \\
\hline 3 (Imer) & $\begin{array}{l}-87.0 \\
(6.7)\end{array}$ & $\begin{array}{l}-64.5 \\
(11.4)\end{array}$ & \multicolumn{3}{|l|}{$\begin{array}{l}-78.5 \\
(7.6)\end{array}$} \\
\hline 4 (MCMCglmm) & $\begin{array}{l}-88.2 \\
(13.0)\end{array}$ & $\begin{array}{l}-35.6 \\
(46.0)\end{array}$ & \multicolumn{3}{|l|}{$\begin{array}{l}-72.4 \\
(18.4)\end{array}$} \\
\hline 5 (MCMCglmm) & $\begin{array}{l}-87.9 \\
(13.6) \\
\end{array}$ & $\begin{array}{l}-12.1 \\
(64.4) \\
\end{array}$ & \multicolumn{3}{|l|}{$\begin{array}{l}-67.4 \\
(22.4)\end{array}$} \\
\hline \multicolumn{4}{|l|}{6 (MCMCglmm) } & $\begin{array}{l}0.193 \\
\left(-1.653^{\mathrm{ns}}, 1.237,0.25\right)\end{array}$ & $\begin{array}{l}0.595 \\
\left(-0.512^{\mathrm{ns}}, 0.905,0.38\right)\end{array}$ \\
\hline \multicolumn{4}{|l|}{7 (MCMCglmm) } & $\begin{array}{l}0.179 \\
\left(-1.720^{n s}, 1.333,0.23\right)\end{array}$ & $\begin{array}{l}0.488 \\
\left(-0.718^{\mathrm{ns}}, 0.812,0.44\right)\end{array}$ \\
\hline \multicolumn{6}{|c|}{$\begin{array}{l}{ }^{b} \text { Predictions of density were obtained by setting } \bar{T}_{i j} \text { to average values of } 3.05 \mathrm{C} \text { and } 0.75 \mathrm{C} \text { for north and south strata, respectively. } \\
{ }^{c} \text { Note that the centred equivalent to } 1985 \text { was used in the calculation but the actual year is shown for ease of interpretation. } \\
\text { [i.e. 1-Prob(Type /I error)] to detect a } 70 \% \text { or areater decline assuming a Gaussian distribution for }\end{array}$} \\
\hline
\end{tabular}

$$
L_{R}(1985,20)
$$




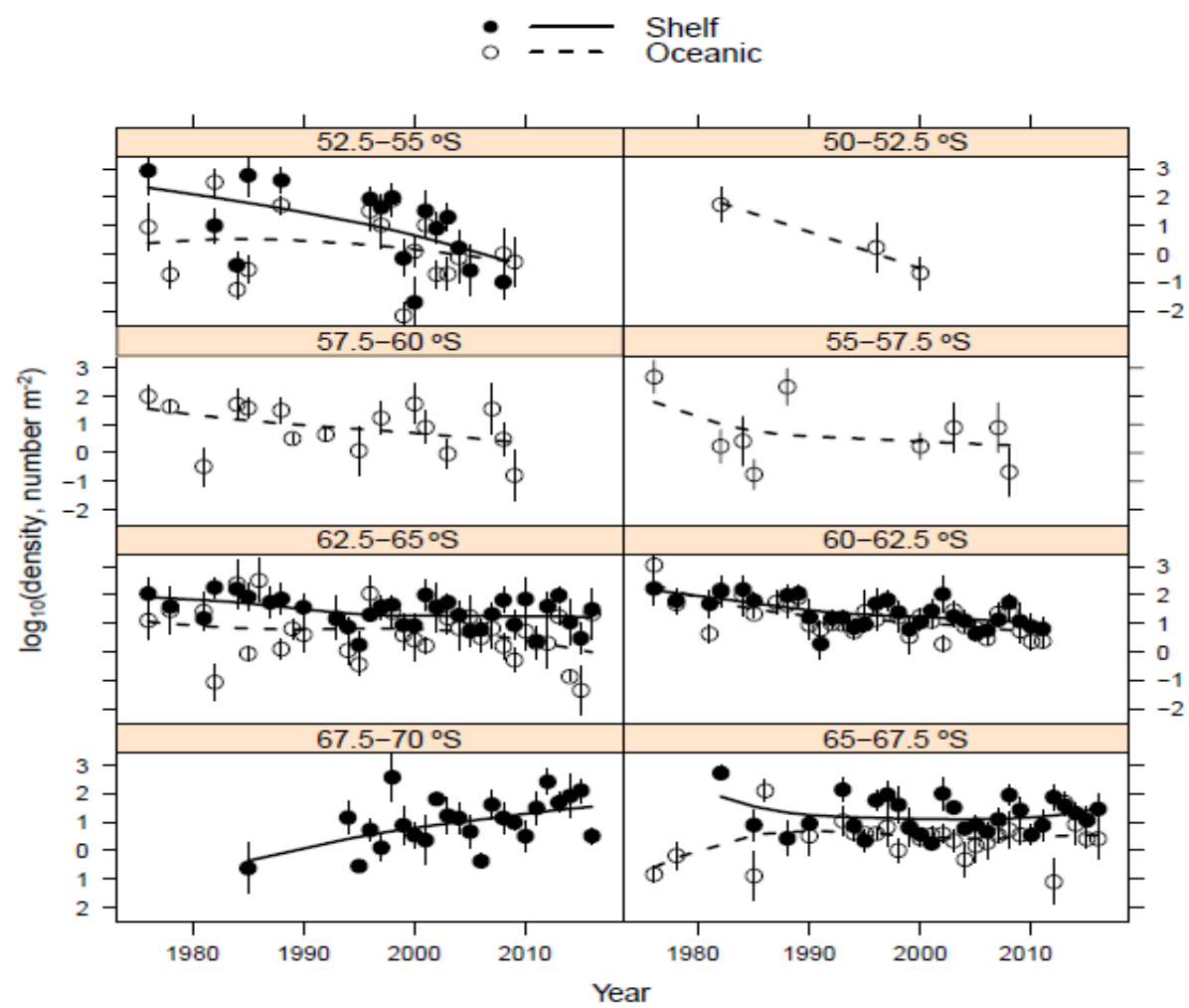

Fig. 1. Spatial cell by year $\log _{10}$ transform of mean densities (showing double SE bars) and fitted splines using MCMCgImm (cf: Fig. 2a of Atkinson et al 2019)

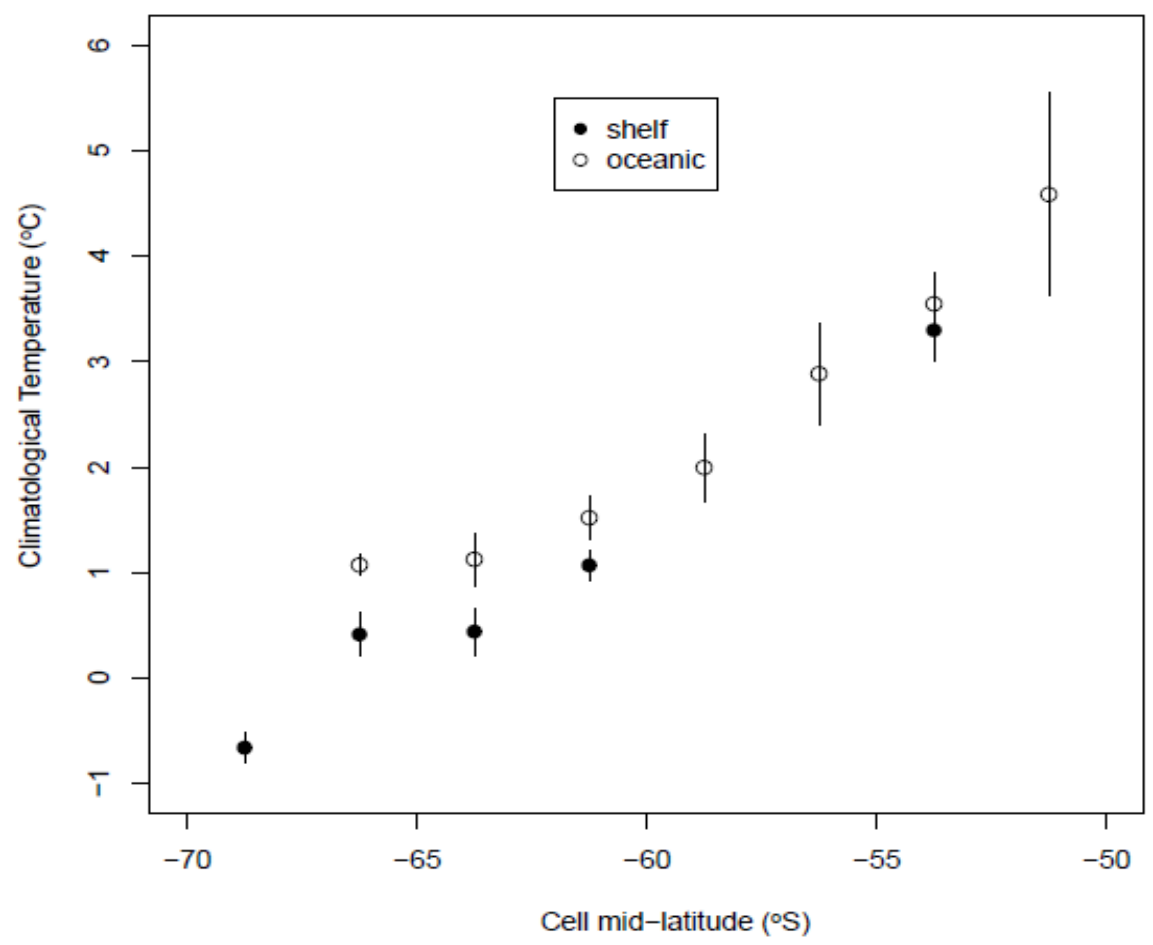

Fig. 2. Mean Climatological Temperature, $\bar{T}_{i}$, versus mid-latitude for each spatial cell (showing double SE bars) 


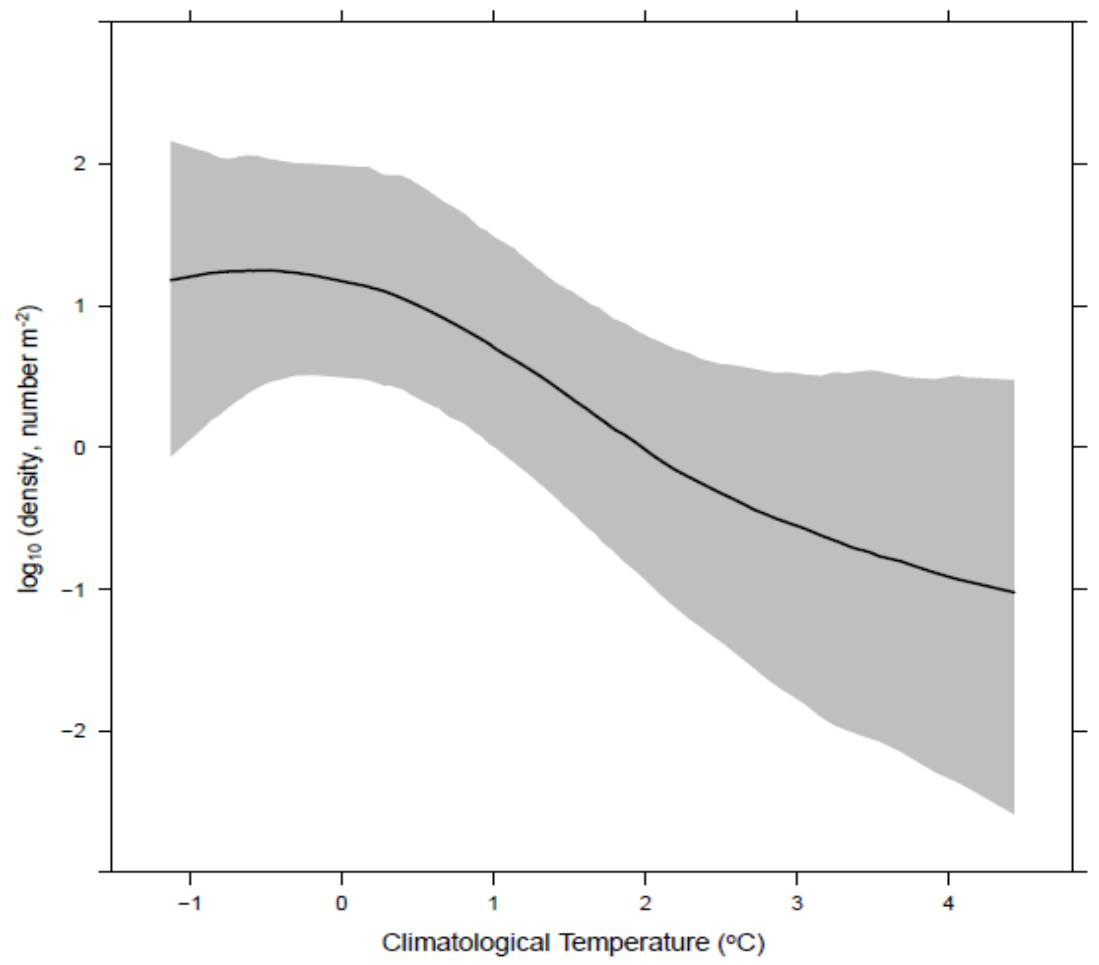

Fig. 3. Predicted relationship between log10 standardised density and Climatological Temperature, $\bar{T}_{i j}$, obtained from Model 7 and $90 \%$ support bounds given centred year, $S$, set to zero

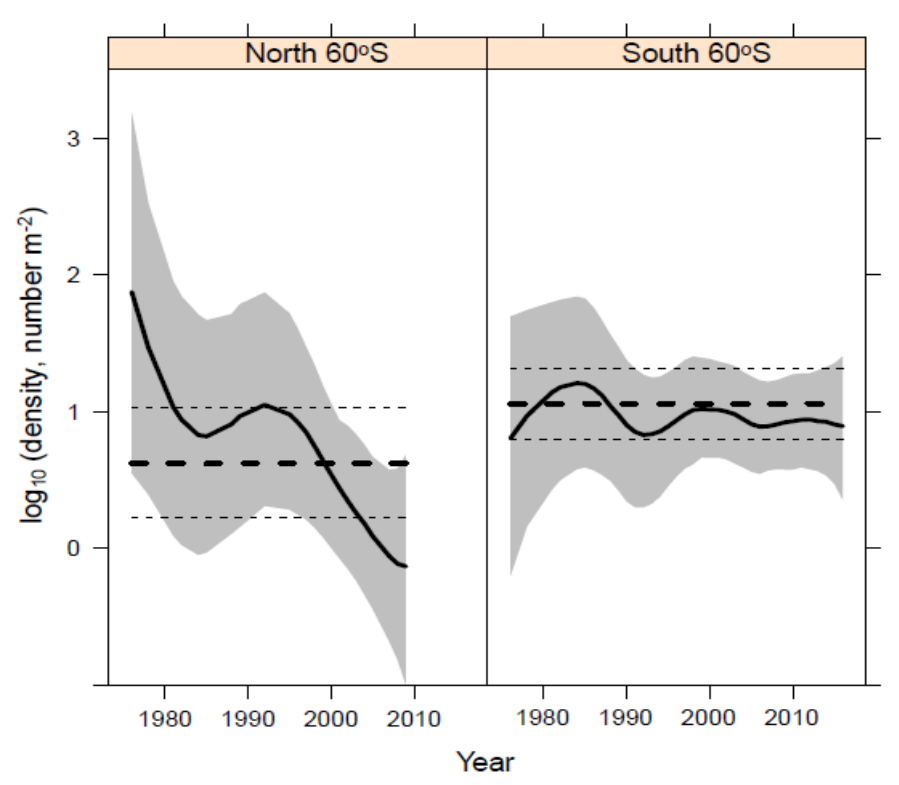

Fig. 4. Median of predicted year trends in log10 standardised density (black lines) and $90 \%$ support bounds (grey fill) using Model 7 for each of north and south strata along with corresponding median predictions for the no-trend model (dashed thick black lines) and $90 \%$ support bounds (dashed thin black lines) obtained for climatological temperature standardised to average values of $3.05^{\circ} \mathrm{C}$ and $0.75^{\circ} \mathrm{C}$ for north and south strata, respectively, with predictions obtained from MCMCgImm samples 
Fig. 3 shows the predicted relationship between log10 standardised density and Climatological Temperature, $\bar{T}_{i j}$, obtained from Model 7 and $90 \%$ support bounds given centred year, $S$, set to zero.

Fig. 4 shows predicted year trend in $\log 10$ standardised density obtained from Model 7 and $90 \%$ support bounds for both north and south regions along with corresponding predictions for the no-trend model each obtained for Climatological Temperature standardised to average values of $3.05^{\circ} \mathrm{C}$ and $0.75^{\circ} \mathrm{C}$ for north and south strata, respectively, with predictions obtained from MCMCglmm fits. The no-trend model corresponds to Model 5 but with all terms in centred year, $S$, dropped and climatological temperature standardised as above.

\section{DISCUSSION}

Model comparisons using AIC and DIC statistics, shown in Table 2, indicate that for the models that assume that the mean densities are a response variable that has no sampling (i.e. "measurement") error (Models 1 to 3 ), that including the random year effect in Model 3 (i.e.

estimating $\sigma_{v}$ ) gave the best (i.e. lowest) AIC. The linear year trends for Model 3, as for Models 1 and 2, were significant and negative for both regions, and in terms of percentage reduction per year or decade, Table 3 estimates of $87 \%$ (North) and 64\% (South) and 78\% (averaged) with standard errors of $7 \%, 11 \%$ and $8 \%$, respectively. These model outputs indicate the dramatic declines touted by Atkinson et al. $[1,3]$. However, when Model 3 was corrected by considering the mean densities, as they are as sample estimates and including the estimated sample variances using Model 4, the statistical significance levels of the linear year-trend coefficients are substantially reduced and the percentage reduction estimate for the Southern region is reduced to $36 \%$ and the standard error is greater than $100 \%$ of the estimate. This effect is greater again when the Climatological Temperature is included as a covariate (Model 5). This was not the case for the Northern region which showed a similar estimated decline for Models 4 and 5 to that of Models 1 to 3 . However, when nonlinearity in the year trends was incorporated in Models 6 and 7, the percentage decline between 1985 and 2005 is no longer statistically significant for either region (Table 3). Since it is not clear from the estimated coefficients in Table 2 what the significance and shape of the year trend for Models 6 and 7 are in terms of departure from a no-trend fit, since the random effect component of each thin-plate spline is quantified in Table 2 as a single variance component, the best way to evaluate the trends is using Fig. 4. Clearly, there is no evidence of a consistent and statistically significant decline for the Southern region with the no-trend line falling well within $90 \%$ support bounds for the spline-predicted year trend. For the Northern region, Fig. 4 indicates a more consistent and substantial decline particularly for pre-1985 and post-1995 periods. However, the $90 \%$ support bounds are much larger, due largely to the large value of $\hat{\sigma}_{N}$ resulting in close to a three times larger estimate of the lack-of-fit error variance (Table 2), and the no-trend line comes close to being enclosed by these bounds. The limitation that MCMCglmm cannot fit versions of Models 4 to 7 that replace their random coefficient error terms with a CAR term, which is the difference between Models 3 and 2, respectively, is not a substantial weakness since by way of comparison Model 1 gave only a slightly higher AIC than Model 2 while Model 3 gave a substantially lower AIC compared to Model 1 . This is the relevant model comparison, rather than comparing Models 1 and 2, since as with Models 4 to 7, Model 3 includes the random year effect $v_{j}$.

Another limitation of the models that include $\bar{T}_{i j}$ as a predictor is that this covariate is subject to sampling error due to the spatial averaging process across hauls and, additionally, due to the across-year averaging used to create this variable in KRILLBASE. Errors-in-variables in linear modelling can result in bias in both point estimates of model parameters and their uncertainty [24]. However, given the relatively small standard errors of the $\bar{T}_{i j}$ as seen in Fig. 2 any biases are likely to be minor; see [25] for an example of an errors-in-variables investigation of sampling error in a covariate due to averaging using Monte Carlo simulation for a generalized linear model where these biases were very small attenuations.

In terms of prediction using Model 7 and how to incorporate $\bar{T}_{i j}$, as noted earlier (see Supplementary Material to Cox et al. [2]) Climatological Temperature in KRILLBASE is a 
long-term (1979-2014) February average of seasurface water temperature for each station (see Table 2 in [6]). Therefore, this component of the year trend in log of mean density, equation (2.3), was "conditioned out" of predictions by setting the value of $\bar{T}_{i j}$ to its centred, simple mean value for each region (as described in Methods). As Cox et al. [2] note, this covariate is fundamentally a nuisance spatial variable since the sampled values corresponding to station locations have a temporal component only because of the order in which stations have been sampled. Therefore, any long-term year trends in $\bar{T}_{i j}$ are an artifact of the imbalance in spatio-temporal sampling and need to be removed (i.e. controlled for) in predicted long-term trend in density as outlined in the general in point (ii) in the Introduction. Note that if this sea temperature variable was potentially informative of the long-term trend in krill density by taking the measured values at the location and time of each station's haul rather than the station's long-term February average, and if significantly predictive of density then its predicted year trend rather than a simple mean should be included in prediction of the year trend in krill density.

\section{CONCLUSIONS}

In conclusion, there is some evidence of a decline in density for the Northern region (above $60^{\circ} \mathrm{S}$ ) but very little for the Southern region (below $60^{\circ} \mathrm{S}$ ). However, uncertainty of predictions using the best model, Model 7, resulted in only weak power to detect a substantial decline of the order of $70 \%$ between 1985 and 2005 with statistical power estimates of 0.23 and 0.44 for the Northern and Southern regions, respectively. For some perspective, experimental design principles suggest statistical power of 0.8 or greater is the recommended target in setting the amount of replication or repeat sampling along with other recommended features of randomisation, adjustment for covariates, elimination of confounding factors, and other application-specific recommendations for desirable experimental or survey design [26]. Therefore, these model-based inferences neither strongly support nor reject a general hypothesis that there has been a dramatic decline in density of Antarctic krill in the Southwest Atlantic over this period.

In terms of the conflicting inferences between Atkinson et al. [3] and Cox et al. [2], this study using a close to matching dataset and a corrected and augmented version of the LMM of Atkinson et al. [3] shows that long-term trend predictions are subject to a very high degree of uncertainty similar to that shown in Fig. 3 of [2]. Cox et al. [2] did not predict separate trends for the northern and southern regions of the south Atlantic as in Atkinson et al. (2019) and here, and while there is evidence of a decline north of $60^{\circ} \mathrm{S}$ and the corresponding inference made by Atkinson et al. [3] that this is associated with a southern contraction in the range of Antarctic krill, the results from Model 7 demonstrate that there is a considerably greater degree of uncertainty as to the magnitude of the decline north of $60^{\circ} \mathrm{S}$ than that inferred from the linear parameter estimate in Atkinson et al. [3]. South of $60^{\circ} \mathrm{S}$ there is a lack of a clear declining trend combined with a substantial degree of uncertainty about the average trend. Therefore, these results suggest, that in the absence of more fit-for-purpose, decadal-level and spatially comprehensive datasets than KRILLBASE, as described by points (i) and (ii) in the Introduction, consideration of long-term year trend predictions using KRILLBASE should carefully evaluate the uncertainty of these predictions. Further, given that the uncertainty of such predictions by Atkinson et al. [3] of a dramatic decline of krill stocks relative to estimated abundance in the mid-1970s has been substantially underestimated and trends have been unnecessarily restricted to a simple linear decline, this study suggests that stakeholders have not been adequately informed of the degree of caution required in evaluating the significance of estimated trends in terms of ecological, commercial (in regard to fishing pressure) and conservation outcomes. As Cox et al. [2] note, predictions of a dramatic decline of the order of $80 \%$ or more in Antarctic krill abundances since the mid-1970s in the Southwest Atlantic should not be unduly influential given their associated uncertainty and given the lack of observation of the expected dramatic negative impact on populations of krill-dependent predators of such a decline.

\section{SUPPLEMENTARY MATERIAL}

Supplementary Material is available in this link: https://www.journalarrb.com/index.php/ARRB/libr aryFiles/downloadPublic/21

\section{ACKNOWLEDGEMENTS}

This work was funded by SCandy Statistical Modelling Pty Ltd. 


\section{COMPETING INTERESTS}

Author has declared that no competing interests exist.

\section{REFERENCES}

1. Atkinson A, Siegel V, Pakhomov EA, Rothery $P$. Long-term decline in krill stock and increase in salps within the Southern Ocean. Nature. 2004;432:100-3.

Available:https://doi.org/10.1038/nature029 96

2. Cox MJ, Candy S, de la Mare WK, Nicol1 S, Kawaguchi S, Gales N. No evidence for a decline in the density of Antarctic krill Euphausia superba Dana, 1850, in the Southwest Atlantic sector between 1976 and 2016. J Crustac Biol. 2018;38:656-61.

3. Atkinson A, Hill SL, Pakhomov EA, Siegel V, Reiss CS, Loeb V, Steinberg DK, Schmidt K, Tarling GA, Gerrish L, Sailley SF. Krill (Euphausia superba) distribution contracts southward during rapid regional warming. Nat Clim Change. 2019;9:1427.

Available:http://dx.doi.org/10.1038/s41558018-0370-z

4. Cochran WG. Sampling Techniques. Third Edition. John Wiley \& Sons, New York; 1977.

5. Särndal CE. Design-based and modelbased inference in survey sampling. Scand J Statist. 1978;5(1):27-52.

6. Atkinson A, Hill SL, Pakhomov EA, Siegel V, Anadon R, Chiba S et al. KRILLBASE: a circumpolar database of Antarctic krill and salp numerical densities, 1926-2016. Earth Syst Sci Data 2017; 9: 193-210.

DOI: 10.5285/8b00a915-94e3-4a04-A903Dd4956346439

7. Maunder MN, Punt AE. Standardizing catch and effort data: a review of recent approaches. Fisheries Res. 2004;70(2-3): 141-59.

Available:https://doi.org/10.1016/j.fishres.2 004.08.002

8. Candy SG. Modelling catch and effort data using generalised linear models, the Tweedie distribution, random vessel effects and random stratum-by-year effects. CCAMLR Sci. 2004;11:59-80.

9. Hill SL, Atkinson A, Pakhomov EA, Siegel V. Evidence for a decline in the population density of Antarctic krill Euphausia superba Dana, 1850 still stands. A comment on Cox et al. J Crustac Biol. 2019;39:316-22.
DOI: $10.1093 / \mathrm{jcbiol} / \mathrm{ruz} 004$

10. Cox MJ, Candy S, de la Mare WK, Nicol1 S, Kawaguchi S, Gales N. Clarifying trends in the density of Antarctic krill Euphausia superba Dana, 1850 in the South Atlantic: a response to Hill et al. J Crustac Biol. 2019;39:323-327.

DOI: $10.1093 / \mathrm{jcbiol} / \mathrm{ruz010}$.

11. Crainiceanu CM, Ruppert D, Wand MP. Bayesian Analysis for Penalized Spline Regression Using WinBUGS. J Stat Softw. 2005;14:1-24.

DOI: $10.18637 /$ jss.v014.i14

12. RCore Team. R: A Language and Environment for Statistical Computing. $R$ Foundation for Statistical Computing, Vienna, Austria; 2013.

13. Draper NR, Smith H. Applied Regression Analysis. Third Edition. John Wiley \& Sons, New York; 1998.

14. Diggle PJ, Heagerty PJ, Liang K, Zeger SL. Analysis of Longitudinal Data, Second Edition. Oxford University Press, Oxford UK; 2002.

15. Pinheiro JC, Bates DM. Mixed-effect Models in S and S-PLUS. Springer, New York; 2004.

16. Hadfield JD. MCMC Methods for MultiResponse Generalized Linear Mixed Models: The MCMCglmm R Package. J Stat Softw. 2010;33(2):1-22

Available:http://www.jstatsoft.org/v33/i02/

17. Bates D, Mächler M, Bolker B, Walker S. Fitting linear mixed-effects models using Ime4. J Stat Softw. 2015;67:1-48.

DOI: $10.18637 /$ jss.v067.i01

18. McCullagh P, Nelder JA. Generalized Linear Models, Second Edition. Chapman and Hall, London; 1989.

19. Goldstein H. Multilevel statistical methods. Second Edition. Edward Arnold, London; 1995.

20. Candy SG, Ziegler P, Welsford DC. A nonparametric model of empirical length distributions to inform stratification of fishing effort for integrated assessments. Fisheries Res. 2014;159:34-44. Available:https://doi.org/10.1016/j.fishres.2 014.05.002

21. Akaike $H$. Information theory and an extension of the maximum likelihood principle. In: Selected papers of Hirotugu Akaike. Springer; 1998.

22. Spiegelhalter DJ, Best NG, Carlin BP, van der Linde A. Bayesian measures of model complexity and fit (with discussion). J Roy Stat Soc B Met. 2002;64:583-640. 
23. Patterson HD, Thompson R. Recovery of interblock information when block sizes are unequal. Biometrika. 1971;58:545-54.

24. Carroll RJ, Ruppert D, Stefanski LA, Crainiceanu CM. Measurement Error in Nonlinear Models: A Modern Perspective, Second Edition. Chapman \& Hall/CRC, New York; 2006.

25. Candy SG. Empirical binomial sampling plans: model calibration and testing using
Williams' method III for generalized linear models with overdispersion. JABES. 2002; 7:373-8.

Available:https://doi.org/10.1198/10857110 2302

26. Gardiner WP, Gettinby GG. Experimental Design Techniques in Statistical Practice: a practical software-based approach. Horwood Publishing, Chichester, England; 1998.

(0) 2021 Candy; This is an Open Access article distributed under the terms of the Creative Commons Attribution License (http://creativecommons.org/licenses/by/4.0), which permits unrestricted use, distribution, and reproduction in any medium, provided the original work is properly cited.

Peer-review history:

The peer review history for this paper can be accessed here: https://www.sdiarticle5.com/review-history/76466 